\title{
Uma Abordagem Evolutiva para o Problema de Custo Médio a Longo Prazo com Saltos Não-Observados
}

C.A. SILVA, D.C. BORTOLIN 2 , E.F. COSTA ${ }^{3}$, Departamento de Matemática Aplicada e Estatística, ICMC/USP - São Carlos, Caixa Postal 668, 13560-970 São Carlos, SP.

\begin{abstract}
Resumo. Neste artigo propomos uma adaptação de um algoritmo baseado na evolução biológica para a obtenção do controle ótimo do problema do custo médio a longo prazo para sistemas lineares com saltos markovianos. Não há na literatura um método que forneça, comprovadamente, o controle ótimo do problema, nem estudos comparativos de diferentes métodos. O algoritmo empregado diferencia-se dos algoritmos genéticos básicos por substituir os operadores evolutivos por um sorteio de acordo com uma distribuição probabilística. Comparamos o algoritmo proposto com um método bastante utilizado para esta classe de problema, levando em consideração a relação entre os custos obtidos, o tempo de CPU e a quantidade de problemas em que o critério de parada estabelecido foi atingido.
\end{abstract}

Palavras-chave. Sistemas com saltos markovianos, algoritmo evolutivo, problema de controle.

\section{Introdução}

Neste artigo tratamos de sistemas lineares com saltos markovianos (SLSM) em tempo discreto [4], os quais representam uma classe de sistemas estocásticos que apresentam modos de operação que se alternam de acordo com as probabilidades de uma cadeia de Markov. Desta forma, estes sistemas apresentam variações repentinas nos parâmetros, chamadas de "saltos". Dizemos que os saltos dos sistemas são observados quando estes são acessíveis em cada instante de tempo, caso contrário são denominados não-observados.

Inúmeras aplicações podem ser modeladas como um SLSM, como controle para aeronaves $[1,15]$, políticas monetárias $[17,2]$, sistemas robóticos [13], entre outros. Além dos aspectos práticos, o fato dos SLSM generalizarem sistemas lineares determinísticos e apresentarem resultados fortes que recuperam propriedades de sistemas lineares clássicos, os torna alvo de trabalhos que abordam um estudo mais teórico.

\footnotetext{
${ }^{1}$ calex@usp.br. Os autores agradecem o apoio financeiro da FAPESP (Proc. 2008/02035-8) e do CNPq (Proc. 135663/2009-1, 306466/2010-4, 135597/2011-0).

2 daiane.bortolin@usp.br

3 efcosta@icmc.usp.br
} 
Destacam-se resultados sobre noções de estabilidade [16], filtragem [14], solução ótima para custo finito e infinito quadrático [7], entre outros. Apesar das várias pesquisas envolvendo os SLSM, ainda existem algumas lacunas, como a determinação do controle ótimo para o custo médio a longo prazo (CMLP). O controle indica uma determinada ação (representada por uma variável) sobre um sistema dinâmico, a fim de "controlar" o sistema. A expressão "controlar", no contexto do artigo, representa encontrar valores para o controle que minimizem o custo representado pelo CMLP.

O CMLP representa o índice de desempenho do SLSM quando há presença de ruído aditivo no sistema, ou seja, quando a trajetória do sistema sofre perturbações, possivelmente de alguma força exógena. Neste contexto, estamos interessados em obter o controle ótimo de um SLSM em que haja presença de ruído aditivo e que não se tenha acesso à informação do estado da cadeia, pois para o cenário onde pode-se observar o estado da cadeia de Markov, a solução ótima é obtida na forma de ganhos de realimentação linear, por meio das soluções das equações algébricas de Riccati (EAR) [4].

No cenário sem observação ou com observação incompleta do estado da cadeia de Markov, onde um único ganho refere-se a todos os estados em cada instante de tempo, não se tem resultados que permitam calcular um controle tal que o CMLP seja um mínimo global. Apenas dispõe-se de um resultado em que se formula uma expressão, assumindo o controle na forma de realimentação linear (compatível com o cenário observado). Em [6] é apresentado um algoritmo para os problemas relacionados aos SLSM sem ruído, o qual foi estendido para o problema do CMLP por [19].

Neste trabalho propomos uma adaptação de um algoritmo evolutivo, Univariate Marginal Distribution Algorithm (UMDA), e comparamos o seu desempenho com o método variacional (MV) desenvolvido por [19], analisando a relação entre os custos obtidos pelos métodos. Em ambos os algoritmos é feita uma aproximação do CMLP via o custo de horizonte finito (CHF) como descrito em [5].

O artigo está organizado da seguinte forma: na Seção 2. são apresentados alguns resultados preliminares e a formulação matemática do problema de otimização, bem como o MV. O algoritmo proposto, UMDA, é detalhado na Seção 3.. Na Seção 4. é apresentado um exemplo que ilustra a aplicabilidade dos SLSM e a utilização dos métodos descritos para o problema do CMLP. Além disso, na Seção 5. é apresentada a comparação do desempenho dos algoritmos implementados. Por fim, conclusões do trabalho encontram-se na Seção 6. .

\section{Notações e Resultados Preliminares}

Seja $\mathcal{S}=\{1, \ldots, S\}$ um conjunto finito e $\mathcal{M}^{r, s}\left(\mathcal{M}^{r}\right)$ a representação de um espaço linear formado por todas as matrizes de dimensão $r \times s(r \times r)$. Definimos $\mathbb{M}^{r, s}=$ $\left\{U=\left(U_{1}, \ldots, U_{S}\right): U_{i} \in \mathcal{M}^{r, s}, i \in \mathcal{S}\right\}$. Denotamos por $U^{\prime}$ a matriz transposta de $U$ e $\operatorname{tr}\{\cdot\}$ o operador traço. O espaço $\mathbb{M}^{r, s}$ equipado com o produto interno, como 
definido abaixo, forma um espaço de Hilbert,

$$
\langle U, V\rangle=\sum_{i=1}^{S} \operatorname{tr}\left\{U_{i}^{\prime} V_{i}\right\}
$$

Seja $(\Omega, \mathcal{F}, P)$ um espaço de probabilidade fundamental. Consideremos $\Theta=$ $\left\{\theta_{k}, k \geq 0\right\}$ uma cadeia de Markov homogênea tal que as probabilidades de transição são dadas por $P\left(\theta_{k+1}=j \mid \theta_{k}=i\right)=p_{i j}$ para todo $i, j \in \mathcal{S}$, com o vetor de distribuição inicial $\pi_{0} \in \mathcal{M}^{1, S}$. Neste contexto, consideramos o SLSM,

$$
\boldsymbol{\Phi}: \begin{cases}x_{k+1}=A_{\theta_{k}} x_{k}+B_{\theta_{k}} u_{k}+G_{\theta_{k}} w_{k}, & \forall k \geq 0, \\ y_{k}=x_{k}^{\prime} C_{\theta_{k}} x_{k}+u_{k}^{\prime} D_{\theta_{k}} u_{k}, & \theta(0) \sim \pi(0),\end{cases}
$$

onde $x_{k} \in \mathcal{M}^{n, 1}$ é usualmente chamado de estado do sistema, $y_{k} \in \mathcal{M}^{1}$ representa um índice de desempenho associado ao estágio $k$ e $u_{k} \in \mathcal{M}^{m, 1}$ é o controle ou ação de entrada no sistema, que pode ser representada por uma variável cujo valor podemos definir ou projetar de forma a minimizar o índice de desempenho. Neste artigo, $w_{k} \in \mathcal{M}^{q, 1}$ representa um ruído, o qual forma um processo aleatório independente e identicamente distribuído (iid) com média zero e covariância $E\left\{w_{k} w_{k}^{\prime}\right\}=\Sigma$, com $E\{\cdot\}$ representando o valor esperado usual.

A cada instante $k$, dado $\theta_{k}=i$, tem-se $A_{\theta_{k}}=A_{i}$ e similarmente para as demais matrizes em (2.1), onde $A_{i} \in \mathcal{M}^{n}, B_{i} \in \mathcal{M}^{n, m}, G_{i} \in \mathcal{M}^{n, q}, C_{i} \in \mathcal{M}^{n}, \operatorname{com} C=C^{\prime} \geq$ 0 , e $D_{i} \in \mathcal{M}^{m}$, com $D=D^{\prime}>0$ (veja o exemplo na Seção 4.).

Note que $x_{k}$ forma um processo estocástico, de tal maneira que $y_{k}$ como definido em (2.1) é uma variável aleatória. Por isso, definimos o custo de horizonte $T$, também chamado de custo de $T$ estágios para o SLSM da seguinte forma:

$$
\mathcal{J}^{T}=\sum_{k=0}^{T-1} E\left\{y_{k}\right\}
$$

Neste artigo consideramos que o controlador tem acesso somente a $x_{k}$ em cada instante de tempo $k$ e a variável $\theta_{k}$ não é observada. Assumimos uma lei de controle na forma de realimentação linear dada por

$$
u_{k}=K x_{k}
$$

pois o ganho estático, $K \in \mathcal{M}^{m, n}$, em geral, é mais simples de implementar que o ganho dependente no tempo $\left(K_{k}\right)$, podendo ser utilizado em várias aplicações.

Para a coleção de matrizes $U \in \mathbb{M}^{n}$ e $V \in \mathbb{M}^{n}$, definimos o operador $\mathcal{T}_{U}: \mathbb{M}^{n} \rightarrow$ $\mathbb{M}^{n}$ tal que

$$
\mathcal{T}_{U, i}(V):=\sum_{j=1}^{S} p_{j i} U_{j} V_{j} U_{j}^{\prime}, \quad \forall i \in \mathcal{S},
$$

onde $\mathcal{T}^{0}(V)=V$ e para $t \geq 1$ temos a recursão $\mathcal{T}^{t}(V)=\mathcal{T}\left(\mathcal{T}^{t-1}(V)\right)$.

A Proposição 2.1 é uma adaptação dos resultados de [4] e apresenta uma expressão determinística que facilita o cálculo do custo de $T$ estágios. 
Proposição 2.1. Seja $X \in \mathbb{M}^{n}, Q \in \mathbb{M}^{n}$ e $\Sigma \in \mathbb{M}^{n}$ definidos por $Q_{i}=Q$ e $\Sigma_{i}=\bar{E}$, para todo $i \in \mathcal{S}$. Então,

$$
\mathcal{J}^{T}(X(0))=\sum_{k=0}^{T-1}\langle X(k), Q\rangle,
$$

onde $X(k)=\mathcal{T}_{\mathbf{A}}^{k}(X(0))+\sum_{\ell=0}^{k-1} \mathcal{T}_{\mathbf{A}}^{k-1-\ell}(\Sigma)$ para todo $i \in \mathcal{S}$ com $X_{i}(0)=\pi_{i}(0) x(0) x(0)^{\prime}$ e $\mathbf{A}_{i}=A_{i}+B_{i} K \in \mathcal{M}^{n}$.

A partir deste resultado, podemos formular o seguinte problema de otimização para o CMLP, cuja variável de interesse é o ganho $K$ do controle a ser determinado,

$$
\min _{K} J=\lim _{T \rightarrow \infty} \sup _{T} \frac{g^{T}(X(0))}{T} .
$$

Quando consideramos o horizonte de tempo infinito $(T=\infty)$, o problema torna-se mais complexo, no sentido que $J$ finito não assegura que o sistema controlado seja estável, como pode ser verificado em [12].

\subsection{Método Variacional}

Em [19] é proposto um método variacional para tratar de problemas de controle de SLSM com ruído aditivo, o qual será descrito de forma breve.

Seja o conjunto $\mathbb{S}^{r}=\left\{U \in \mathbb{M}^{r}: U_{i}=U_{i}^{\prime} \geq 0, i \in \mathcal{S}\right\}$ a representação do espaço linear formado pelas matrizes semi-definidas positivas. Definimos os operadores $\mathscr{E}, \mathcal{L}: \mathbb{S}^{n} \rightarrow \mathbb{S}^{n}$, para todo $i \in \mathcal{S}$, tal que

$$
\begin{aligned}
& \mathscr{E}_{i}(\phi)=\sum_{j=1}^{S} p_{i j}, \\
& \mathcal{L}_{i}(k)=C_{i}+g_{k}^{\prime} D_{i} g_{k}+\left(A_{i}+B_{i} g_{k}\right)^{\prime} \mathscr{E}_{i}(\mathcal{L}(k+1))\left(A_{i}+B_{i} g_{k}\right),
\end{aligned}
$$

onde $\phi \in \mathbb{S}^{n}$ e $\mathcal{L}_{i}(T)=0$.

O Teorema 2.1 apresenta uma condição de otimalidade sob o ganho $g_{k} \in \mathcal{M}^{m, n}$.

Teorema 2.1. [19] Suponha que a sequência de ganhos $\mathbf{g}=\left\{g_{0}, \ldots, g_{T-1}\right\}$ fornece o mínimo global do problema do custo $T$ estágios. Então $\mathbf{g}$ satisfaz, para cada $k=0, \ldots, T-1$,

$$
\sum_{i=1}^{S}\left[\left(D_{i}+B_{i}^{\prime} \mathscr{E}(\mathcal{L}(k+1)) B_{i}\right) g_{k}+B_{i}^{\prime} \mathscr{E}(\mathcal{L}(k+1)) A_{i}\right] X_{i}(k)=0 .
$$

Observação 1. Em [6] é apresentado um método para obter a solução da equação algébrica (2.3).

No Algoritmo 1 está apresentado o método variacional que, de acordo com [18], determina o CMLP pela seguinte aproximação: $\mathcal{J}^{T} / T \rightarrow J$ quando $T \rightarrow \infty$, onde $K=g_{T / 2}$. Além disso, pode-se garantir por [18] que a sequência de ganhos $\mathbf{g}=$ $\left\{g_{0}, \ldots, g_{T-1}\right\}$ obtida pelo Algoritmo 1 é um ponto de mínimo local para o problema de controle de $T$ estágios. 
Algoritmo 1 Método Variacional

Passo 0: Inicie o processo com $\eta=0$. Gere aleatoriamente uma sequência inicial de ganhos $\mathbf{g}^{(\eta)}$.

Passo 1: Determine $X^{(\eta)}(k)$. Faça $\eta=\eta+1$. Para cada $k=T-1, T-2, \ldots, 0$, determine $g_{k}^{(\eta)}$ resolvendo $(2.3)$ e calcule $\mathcal{L}^{(\eta)}$ pela equação $(2.2)$.

Critério de parada: $\left|\mathcal{J}^{T}\left(X(0), \mathbf{g}^{(\eta)}\right)-\mathcal{J}^{T}\left(X(0), \mathbf{g}^{(\eta-1)}\right)\right| / \mathcal{J}^{T}\left(X(0), \mathbf{g}^{(\eta-1)}\right)<\epsilon$, para $\epsilon \in \mathbb{R}^{+}$dado. Se o critério não for satisfeito, retorne a $(1)$.

Passo 2: $J_{V}=\mathcal{J}^{T}\left(X(0), g_{T / 2}^{(\eta)}\right) / T$.

\section{Método UMDA}

O método UMDA, proposto por [10], é um algoritmo de estimação de distribuição no qual não existem os operadores de cruzamento e mutação como nos algoritmos genéticos convencionais. Além disso, este método assume que as variáveis são independentes entre si. Em cada geração do algoritmo e para cada variável, buscamos a função densidade de probabilidade normal que melhor representa esta variável. Para isso utilizamos os valores da média e do desvio padrão do conjunto de elementos da população compostos pelas variáveis de cada ganho.

Para gerar a população inicial utilizamos a solução da EAR para o cenário observado. Esta estratégia foi adotada com sucesso em um algoritmo evolutivo aplicado ao SLSM como descrito em [12]. Assim, a partir dos ganhos ótimos $L_{1}, \ldots, L_{S}$ da EAR, podemos definir a população inicial do UMDA por $K^{\ell}=$ $\alpha_{1} L_{1}+\ldots+\alpha_{S} L_{S}, \quad \ell=1, \ldots, n_{p}$, onde $n_{p}$ é o tamanho da população inicial e $\alpha_{i}, i \in \mathcal{S}$, são variáveis gaussianas iid com média zero e matriz de covariância arbitrária. Além disso, para garantir a variabilidade genética, cada elemento do ganho é dado por uma função $f: \mathbb{R}^{p} \rightarrow \mathbb{R}$,

$$
K^{\ell}(i, j)=f\left(\beta_{1}^{\ell}, \ldots, \beta_{p}^{\ell}\right)
$$

onde $p$ é a quantidade de genes de cada cromossomo. Para a inicialização de cada elemento da população, $K^{\ell}$, geramos aleatoriamente $\beta_{1}^{\ell}(i, j), \ldots, \beta_{p-1}^{\ell}(i, j)$ com distribuição uniforme entre -1 e 1 , e definimos $\beta_{p}^{\ell}(i, j)=K^{\ell}(i, j)-f\left(\beta_{1}^{\ell}(i, j)^{1}+\ldots+\right.$ $\left.\beta_{p-1}^{\ell}(i, j)^{p-1}\right)$.

Neste artigo, adotamos $f$ da Equação 3.1 na forma polinomial, pois deste modo é possível obter uma quantidade maior de genes, o que, apesar de levar a um maior custo computacional, também acarreta uma maior variabilidade genética e consequentemente permite explorar o espaço de soluções.

Para a seleção dos melhores indivíduos, os quais representam os ganhos do controle associados aos menores valores do CMLP, utilizamos o conhecido método de Torneio [8]. Este método oferece a vantagem de não exigir a comparação entre todos os indivíduos da população e a de não-geração de "super-indivíduos" [11]. 
Através dos $N$ ganhos selecionados pelo método do torneio, $K^{1}, \ldots, K^{N}$, calculamos a média e desvio padrão pelas equações

$$
\mu_{i, j}=\sum_{\ell=1}^{N} \frac{K^{\ell}(i, j)}{N} \text { e } \sigma_{i, j}=\sqrt{\frac{\sum_{\ell=1}^{N}\left(K^{\ell}(i, j)-\mu_{\ell, j}\right)^{2}}{N}} .
$$

A nova população (novos ganhos $K^{\ell}$ ) são gerados por uma função densidade de probabilidade normal, $K^{\ell}(i, j) \sim N\left(\mu_{i, j}^{\ell}, \sigma_{i, j}^{\ell}\right)$. O procedimento do UMDA é apresentado no Algoritmo 2.

\section{Algoritmo 2 Método UMDA}

Passo 0: Inicie o processo com o número de gerações $n_{g}=0$, e com o contador de gerações sem melhora do custo $c=0$. Determine a população inicial, $P o p=\left\{K^{\ell}, \ell=1, \ldots, n_{p}\right\}$, de acordo com (3.1). Faça $F=K_{n_{g}}^{j}$ tal que $J\left(K_{n_{g}}^{j}\right) \leq J\left(K_{n_{g}}^{\ell}\right), \forall \ell$.

Passo 1: Selecione os indivíduos pelo método do Torneio [8]. Calcule $\mu_{\ell, j}$ e $\sigma_{\ell, j}^{2}$ por (3.2) e atualize $P o p=\left\{K^{\ell}, \ell=1, \ldots, N\right\}$.

Passo 2: Determine o ganho $\bar{F}=K_{n_{g}}^{j}$ tal que $J\left(K_{n_{g}}^{j}\right) \leq J\left(K_{n_{g}}^{\ell}\right), \forall \ell$.

Passo 3: Se $J(\bar{F})<J(F)$ então $F=\bar{F}$ e $c=0$, caso contrário, $c=c+1$. Faça $n_{g}=n_{g}+1$.

Critério de parada: $n_{g}<c$. Se o critério não for satisfeito, retorne a (1).

Passo 4: $J_{U}=J(F)$.

\section{Exemplo Ilustrativo}

Nesta seção apresentamos um exemplo que consiste em uma adaptação de [3], que ilustra a aplicação do SLSM e a utilização dos métodos variacional e UMDA.

Considere um sistema de produção de uma indústria que fabrica dois tipos de produtos. A demanda por estes produtos no tempo $k$ será representada por um sequência de vetores bi-dimensionais aleatórios $\left\{w_{k}, k \geq 0\right\}$ iid para todo $k$. A indústria deseja que a sua produção satisfaça toda a demanda pelos produtos. No entanto, devemos considerar que o sistema de produção está sujeito a falhas, e portanto, podemos ter dois possíveis estados de Markov: sistema de produção operando (estado 1) ou não operando (estado 0). Podemos representar este processo pelo seguinte diagrama (Figura 1), onde estão apresentadas as probabilidades de transição de cada estado:

Sejam $x_{k}, u_{k}$ e $w_{k}$, o estoque, a produção total e a demanda dos produtos no tempo $k$, respectivamente. Logo, temos que o estoque do produto no tempo $k+1$ 


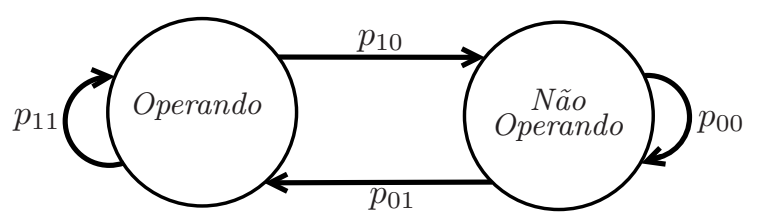

Figura 1: Diagrama de estados da cadeia de Markov.

é dado por

$$
x_{k+1}=A_{\theta_{k}} x_{k}+B_{\theta_{k}} u_{k}+G_{\theta_{k}} w_{k},
$$

onde $A_{0}=A_{1}=I^{2}, G_{0}=G_{1}=-I^{2}$, onde $I^{n}$ denota a matriz identidade de dimensão $n \times n$, e $B_{0}=\left[\begin{array}{ll}0 & 0\end{array}\right]^{\prime}$ e $B_{1}=\left[\begin{array}{ll}1 & 1\end{array}\right]^{\prime}$. A matriz de transição desse processo é denotada por $\mathbb{P}=\left[p_{i j}\right], i, j=0,1$, com $p_{i j} \in(0,1)$. O problema consiste em controlar a produção $u_{k}$ no tempo $k$ de modo a minimizar o custo da produção

$$
y_{k}=x_{k}^{\prime} C_{\theta(k)} x_{k}+u_{k}^{\prime} D_{\theta(k)} u_{k},
$$

onde $C_{0}=C_{1}=\rho^{k} r I^{2}$ e $D_{0}=D_{1}=\rho^{k} \operatorname{com} r>0$ e $\rho \in(0,1)$.

De acordo com [3] podemos reescrever (4.1) e (4.2) de modo a obter um problema equivalente com os seguintes parâmetros para o SLSM: $A_{0}=A_{1}=\rho^{0,5} I^{2}, G_{0}=$ $G_{1}=-\rho^{0,5} I^{2}, B_{0}=\left[\begin{array}{ll}0 & 0\end{array}\right]^{\prime}, B_{1}=\rho^{0,5}\left[\begin{array}{ll}1 & 1\end{array}\right]^{\prime}, C_{0}=C_{1}=r I^{2}$ e $D_{0}=D_{1}=1$. Aplicaremos os métodos variacional e $\mathrm{UMDA}^{4}$ no SLSM equivalente para encontrar uma aproximação para o problema do CMLP. Para tal, consideramos os seguintes parâmetros:

$$
r=0,8, \rho=0,99, x_{0}=\left[\begin{array}{ll}
0 & 0
\end{array}\right]^{\prime}, \pi(0)=\left[\begin{array}{ll}
0,8066 & 0,1934
\end{array}\right] \text { e } \mathbb{P}=\left[\begin{array}{cc}
0,1 & 0,9 \\
0,05 & 0,95
\end{array}\right] .
$$

O MV foi iniciado com a sequência de ganhos $\mathbf{g}^{(0)}$ tal que $g_{k}^{(0)}=\left[\begin{array}{ll}0 & 0\end{array}\right]$, para todo $k=0, \ldots, T-1$. Já o UMDA, foi iniciado com os ganhos $L_{1} \approx\left[\begin{array}{ll}0 & 0\end{array}\right] \mathrm{e}$ $L_{2} \approx[-0,3515-0,3515]$, obtidos pela EAR. Além disso, consideramos a função $f: \mathbb{R}^{p} \rightarrow \mathbb{R}$ dada em (3.1) na forma polinomial

$$
K^{\ell}(i, j)=f\left(\beta_{1}^{\ell}, \ldots, \beta_{p}^{\ell}\right)=\beta_{1}^{\ell}(i, j)^{1}+\beta_{2}^{\ell}(i, j)^{2}+\ldots+\beta_{p}^{\ell}(i, j)^{p} .
$$

Visto que para cada cada $\beta_{i}^{\ell}$ temos um efeito com potência $i$ na composição do ganho $K$, o que na prática mostrou ser eficiente em termos de desempenho do método.

O ganho obtido pelo UMDA para $T=100$ é $K_{U} \approx\left[\begin{array}{ll}-0,1950 & -0,2139] \mathrm{com}\end{array}\right.$ custo associado $J_{U} \approx 51,1664$. O MV obteve um ganho $K_{V} \approx\left[\begin{array}{ll}-0,3468-0,3468] & -1\end{array}\right.$ com custo associado $J_{V} \approx 51,1184$.

Na Figura 2 estão apresentados os CMLP obtidos para cada horizonte de tempo $T$. Note que conforme o horizonte aumenta o valor do custo converge, permanecendo constante, ou tendo pequenas variações ao longo do tempo.

\footnotetext{
${ }^{4} \mathrm{O}$ método variacional (Algoritmo 1) e o método UMDA (Algoritmo 2) foram implementados no software $M A T L A B^{\circledR}$
} 


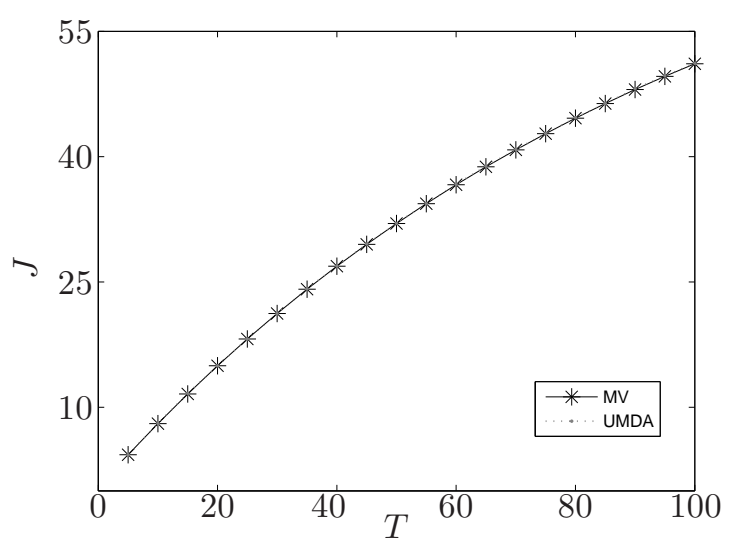

Figura 2: CMLP ao longo do horizonte $T$.

\section{Experimentos Computacionais}

Para avaliar a eficiência dos métodos apresentados, utilizamos um conjunto de problemas testes com 1000 SLSM, cujos parâmetros foram gerados aleatoriamente.

Nos Algoritmos 1 e 2 aproximamos o CMLP via o CHF, ou seja, consideramos um horizonte de tempo finito $T$ suficientemente grande para aproximar o problema de CMLP. A determinação do horizonte $T$ para cada SLSM foi feita da seguinte forma: seja $\tilde{X} \in \mathbb{S}^{n}$ a trajetória do sistema associada à coleção de ganhos $L$ obtida pela EAR e $\bar{\epsilon}$ um escalar suficientemente pequeno. Para cada $k=0,1, \ldots, \bar{k}$, determinamos o valor de $\tilde{X}(k)$ até que este satisfaça a condição $\|\tilde{X}(\bar{k}+1)-\tilde{X}(\bar{k})\| \leq \bar{\epsilon}$, considerando $T=2 \bar{k}$. Além disso, utilizamos o horizonte $T$ para calcular o custo obtido pelo UMDA e $2 T$ para calcular o custo do MV, pois a solução deste método considera o ganho no instante $2 T / 2=T$.

Analisando os dados obtidos pelo MV temos que para 846 problemas, o Algoritmo 1 foi executado e atendeu ao critério de parada estabelecido dentro de um número máximo de iterações (fixado em 500). Para os dados obtidos pelo método UMDA temos que todos os problemas atenderam o critério de parada estabelecido, sendo que o número de gerações sem melhora do custo foi fixado em $c=50$. Assim, as comparações entre os métodos foram realizadas considerando apenas os 846 problemas em que ambos os métodos atingiram o critério de parada.

A Figura 3(a) mostra o custo do MV em relação ao custo do UMDA. Na Figura 3(b) é apresentado o tempo de CPU do MV, $T_{V}$, em relação ao tempo do UMDA, $T_{U}$. Pode ser observado que a partir desses gráficos não podemos afirmar qual dos métodos obteve o melhor desempenho. Desse modo, utilizamos o teste de hipótese [9] para formular uma suposição sobre a média da razão dos custos e outra sobre a média da razão dos tempos de CPU considerando os 846 SLSM.

Teste de Hipótese 1. Deseja-se testar se a média $\bar{\mu}$, razão entre custo em escala logarítmica do $M V$ e do UMDA, é igual a 1, contra a alternativa de ser maior que 


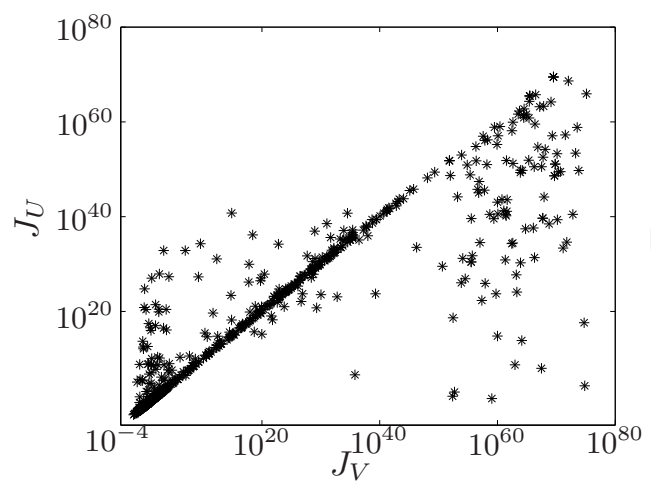

(a) Custo do MV versus o do UMDA.

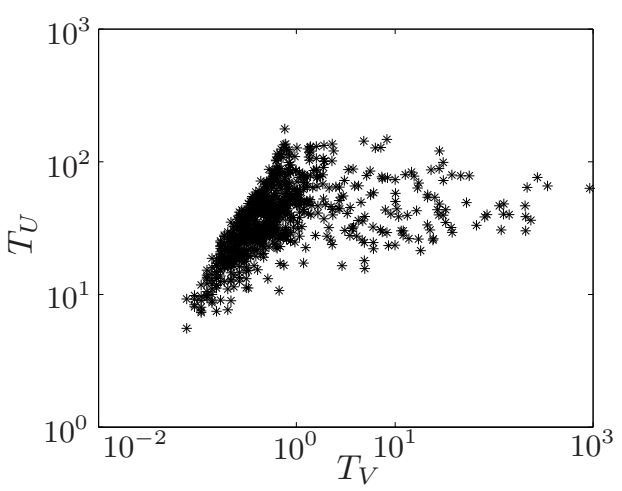

(b) Tempo do MV versus o do UMDA.

Figura 3: Relação entre os dados obtidos.

1. Inferimos através das amostras (custos obtidos) que o desvio padrão do custo é dado por $\sigma \approx 1,7727$. As duas hipóteses sobre a média da amostra são denotadas por $H_{0}$ (Hipótese Nula) e $H_{a}$ (Hipótese Alternativa), respectivamente. Assim,

$$
H_{0}: \bar{\mu}=1 \quad\left(J_{V} \leq J_{U}\right) \quad H_{a}: \bar{\mu}>1 \quad\left(J_{V}>J_{U}\right) .
$$

$O$ erro ao rejeitar a hipótese $H_{0}$ quando, na realidade, $H_{0}$ é verdadeira é dado por

$$
\alpha=P\left[\text { rejeitar } H_{0} \mid H_{0} \text { verdadeira }\right]=P\left[\bar{X}>x_{c} \mid H_{0}\right]=P\left[Z>\frac{x_{c}-\bar{\mu}}{S}\right],
$$

onde $Z=\frac{\bar{X}-\bar{\mu}}{S}$ com $Z \sim N(0,1)$ e $S=\frac{\sigma}{\sqrt{846}}$ (estamos aproximando a distribuição de $Z$ por uma normal pois o número de amostras é grande). Logo, considerando o valor crítico $x_{c}$ igual a 1,02 , temos

$$
\alpha=P\left[Z>\frac{0,02}{0,0609}\right] \approx P[Z>0,3282] \approx 37,13 \% .
$$

$O$ valor obtido para a média foi $\bar{\mu}=1,1273$, de forma que $\bar{\mu}>x_{c}$ implica em rejeitar a Hipótese Nula e concluir que o UMDA obteve custos menores que o $M V$. A probabilidade de estarmos enganados nesta conclusão é de $\alpha=37,13 \%$.

Pelo Teste de Hipótese 1 verificamos que $J_{U}<J_{V}$ com nível de significância $\alpha=37,13 \%$. Para uma análise mais detalhada deste fato, dividimos o conjunto de resultados em duas classes, sendo que a primeira é formada pelos problemas em que $J_{V}<10^{40}$ e a segunda pelos problemas em que $J_{V} \geq 10^{40}$. De acordo com [4] o sistema (2.1) é MS-estável se, e somente se, o raio espectral do operador $\mathcal{T}_{A}$ é menor que 1. Caso contrário, consideraremos o sistema instável. Analisando a MS-estabilidade para a primeira classe, constatamos que $54,36 \%$ dos problemas são estáveis e desse total para apenas 2,67\% temos que o UMDA obteve custos menores. 
Para os 45,64\% problemas instáveis temos que o UMDA obteve custos menores para $5,18 \%$. Para a segunda classe, $100 \%$ dos problemas são instáveis, sendo que para $51,01 \%$ temos que $J_{U}<J_{V}$.

Teste de Hipótese 2. Deseja-se testar se a média $\bar{\mu}$, razão entre tempo de CPU do $M V$ e o tempo do UMDA, é igual a 0,1, contra a alternativa de ser maior que 0,1 . Inferimos através das amostras (tempos obtidos) que o desvio padrão do tempo é dado por $\sigma \approx 0,7461$. As duas hipóteses sobre a média da amostra são denotadas por $H_{0}$ e $H_{a}$, respectivamente. Assim,

$$
H_{0}: \bar{\mu}=0,1\left(T_{U}=10 T_{V}\right) \quad H_{a}: \bar{\mu}>0,1\left(T_{U}>10 T_{V}\right) .
$$

$O$ erro ao rejeitar a hipótese $H_{0}$ quando, na realidade, $H_{0}$ é verdadeira é dado por

$$
\alpha=P\left[\text { rejeitar } H_{0} \mid H_{0} \text { verdadeira }\right] \approx P[Z>0,3898] \approx 34,83 \%,
$$

onde $Z=\frac{\bar{X}-\bar{\mu}}{S} \operatorname{com} Z \sim(0,1)$ e $S=\frac{\sigma}{\sqrt{846}}$ (estamos aproximando a distribuição de $Z$ por uma normal pois o número de amostras é grande) e considerando o valor crítico $x_{c}$ igual a 0,11 . O valor obtido para a média foi $\bar{\mu}=0,1311$, de forma que $\bar{\mu}>x_{c}$ implica em rejeitar a Hipótese Nula e concluir que a o tempo de CPU do UMDA é maior que 10 vezes o tempo de CPU do MV. A probabilidade de estarmos enganados nesta conclusão é de $\alpha=34,83 \%$.

\section{Conclusões}

Neste artigo apresentamos dois métodos aplicáveis ao problema de custo médio a longo prazo para sistemas lineares com saltos markovianos sem observação da cadeia de Markov, onde um é baseado na metodologia dinâmica variacional e o outro na metodologia heurística evolutiva.

O algoritmo evolutivo UMDA difere-se dos algoritmos genéticos básicos por não possuir os operadores de cruzamento e mutação, sendo substituídos por um sorteio de acordo com uma distribuição probabilística. Para este método, propomos uma representação polinomial para os ganhos (genes) de controle, permitindo uma maior variabilidade genética e, consequentemente, uma maior exploração do espaço de soluções. Para avaliar o desempenho deste algoritmo na obtenção do controle ótimo do problema de custo médio a longo prazo, comparamos-o com um método clássico da literatura, método variacional, sendo que na implementação consideramos uma aproximação do custo de horizonte finito para empregar o custo médio a longo prazo.

Os resultados obtidos nos experimentos computacionais (Seção 5.), considerando apenas os problemas em que o método variacional atendeu o critério de parada, sugerem que para um percentual de problemas o UMDA despende um tempo computacional maior quando comparado com o método variacional (Teste de Hipótese 2 ). No entanto, para uma parcela significativa de problemas os custos obtidos pelo UMDA é menor que os obtidos pelo método variacional (Teste de Hipótese 1), principalmente para os problemas que consideramos como instáveis. Este fato indica que o método variacional pode não ter um bom desempenho em situações em que é muito difícil, numericamente, encontrar uma sequência de ganhos inicial com custos relativamente baixos. 


\begin{abstract}
In this paper we propose an adaptation of an algorithm based on biological evolution to obtain optimal control for the long run average cost problem for Markov jump linear systems. There is no in the literature a method that provides, proven, the optimal control of the problem, nor comparatives studies of different methods. The algorithm employed differs from the genetic algorithms to replace the basic operators for rolling a drawing according to a probability distribution. Comparing the proposed algorithm with a widely used method for this class of problem, leading into account the cost obtained, CPU time and amount of problems in which the stopping criterion set has been reached.
\end{abstract}

Keywords. Markov jump systems, evolutive algorithm, control problem.

\title{
Referências
}

[1] L. Blackmore, M. Ono, A. Bektassov, B.C. Williams, A probabilistic particlecontrol approximation of chance-constrained stochastic predictive control. IEEE Transactions on Robotics, 26, No. 3 (2010), 502-517.

[2] O.L.V. Costa, M.V. Araújo, A generalized multi-period mean-variance portfolio optimization with Markov switching parameters. Automatica, 44, No. 10 (2008), 2487-2497.

[3] O.L.V. Costa, J.B.R. do Val, Jump LQ-optimal control for discrete-time markovian systems with stochastic inputs. Stochastic Analysis and Applications, 16, No. 5 (1998), 843-858.

[4] O.L.V. Costa, M.D. Fragoso, R.P. Marques, "Discrete-Time Markovian Jump Linear Systems", Springer-Verlag, New York, 2005.

[5] E.F. Costa, A.N. Vargas, J.B.R. do Val, Quadratic costs and second moments of jump linear systems with general Markov chain, Mathematics of Control, Signals and Systems, 23, No. 1 (2011), 141-157.

[6] J.B.R. do Val, T. Başar, Receding horizon control of jump linear systems and a macroeconomic policy problem, Journal of Economic Dynamics \& Control, 23 (1999), 1099-1131.

[7] Z. Gajic, R. Losada, Solution of the state-dependent noise optimal control problem in terms of Lyapunov iterations, Automatica, 35, No. 5 (1999) 951954.

[8] D.E. Goldberg, "Genetic Algorithms in Search, Optimization and Machine Learning", 1st ed., Addison Wesley, 1989.

[9] M.N. Magalhães, A.C.P. Lima, "Noções de Probabilidade e Estatística", Editora da Universidade de São Paulo, 2002.

[10] H. Muhlenbeing, The equation for response to selection and its use for prediction, Evolutionary Computation, 5, No. 3 (1997), 303-346. 
[11] M. Scheffer, J.M. Baveco, D.L. DeAngelis, K.A. Rose, E.H. van Nes, Superindividuals a simple solution for modelling large populations on an individual basis, Ecological modelling, 80 No. 2-3 (1995), 161-170.

[12] C.A. Silva, E.F. Costa, An algorithm for the long run average cost problem for linear systems with non-observed Markov jump parameters, "American Control Conference", pp. 4434-4439, St. Louis, USA, 2009.

[13] A.A.G. Siqueira, M.H. Terra, T.B.R. Francisco, Controle robusto de robos móveis em formação sujeitos a falhas, Sba Controle $\&$ Automação, 21, No. 1 (2010), 29-42.

[14] C.E. de Souza, M.D. Fragoso, $H_{\infty}$ filtering for discrete-time linear systems with Markovian jumping parameters, International Journal of Robust and Nonlinear Control, 13 (2003) 1299-1316.

[15] P. Stoica, I. Yaesh, Jump Markovian-based control of wing deployment for an uncrewed aircraft, Journal of Guidance, Control, and Dynamics, 25, No. 2 (2002), 407-411.

[16] M.G. Todorov, M.D. Fragoso, On the stability radii of continuous-time infinite Markov jump linear systems, Mathematics of control, Signals, and Systems, 22, No. 1 (2010), 23-38.

[17] A.N. Vargas, J.B.R. do Val, E.F. Costa, Controle de horizonte retrocedente de sistemas lineares com saltos Markovianos para o problema de rastreamento com alvos dinâmicos, SBA Controle $E$ Automação, 16, No. 4 (2006), 435-448.

[18] A.N. Vargas, "Estabilidade e Controle com Critério de Custo Médio a Longo Prazo em Sistemas Lineares Estocásticos", Tese de Doutorado, FEEC, Unicamp, Campinas, SP, 2009.

[19] A.N. Vargas, J.B.R. do Val, E.F. Costa, Receding horizon control of Markov jump linear system subject to noise and unobserved state chain, "IEEE Conference on Decision and Control", pp. 4381-4386, Atlantis, Bahamas, 2004. 